\title{
Hétéromutilation pénienne : à propos d'un cas à la clinique universitaire d'urologie-andrologie du centre national hospitalier universitaire Hubert-Koutoukou-Maga (CNHU HKM) de Cotonou (Bénin)
}

\author{
Heteromutilation of penis: report of a case at the University Teaching Hospital Clinic of Urology- \\ Andrology at CNHU HKM of Cotonou (Benin)
}

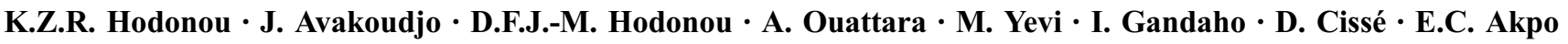

Reçu le 16 juillet 2011 ; accepté le 22 novembre 2011

(C) SALF et Springer-Verlag France 2012

Résumé Traumatismes rares, les amputations du pénis sont le plus souvent retrouvées dans le cadre d'automutilation chez des patients présentant un problème psychiatrique. Nous rapportons un cas d'amputation traumatique du pénis par agression physique. La section du pénis est située à $3 \mathrm{~cm}$ de sa base avec le segment sectionné non retrouvé. La prise en charge du patient a été double. Dans un premier temps, en urgence il a été réalisé une chirurgie d'hémostase, de régularisation du moignon et de mise en place d'une sonde urinaire, puis d'une prise en charge psychologique et psychiatrique dans un second temps. L'évolution est favorable avec une bonne cicatrisation du moignon et une amélioration de l'état psychiatrique sous antidépresseur. Des méthodes de chirurgie reconstructrice, telles les greffes, pourraient être envisagées. Pour citer cette revue : Andrologie 22 (2012).

Mots clés Amputation · Traumatisme · Verge - Agression · Adultère

\begin{abstract}
Amputations of the penis are rare injuries, most often found in self-mutilation where patients have psychiatric problem. We report a case of traumatic amputation of the penis by physical aggression received at the University Teaching Hospital Clinic of Urology-Andrology CNHU HKM Cotonou. The section of the penis is located $3 \mathrm{~cm}$ from the base. The amputated segment is not found. The management of the patient was double. Initially, it was urgently performed surgery to stop bleeding, stabilize the stump and pose of a urinary catheter and a psychiatric treatment at a later time. The evolution is favorable, with good healing of the stump
\end{abstract}

K.Z.R. Hodonou · J. Avakoudjo · D.F.J.-M. Hodonou •

A. Ouattara $\cdot$ M. Yevi $\cdot$ I. Gandaho $\cdot$ D. Cissé $(\bowtie) \cdot$ E.C. Akpo

Centre national hospitalier universitaire Hubert Koutoukou

MAGA de Cotonou, BP 386, Bénin

e-mail : djennecisse@yahoo.fr and an improvement in psychiatric condition as antidepressant. Methods of reconstructive surgery such transplants could be considered. To cite this journal: Andrologie 22 (2012).

Keywords Amputation · Injuries · Penis · Attack · Adultery

\section{Introduction}

Les traumatismes des organes génitaux externes sont rares au regard des autres traumatismes. Plus rares encore, les amputations traumatiques de la verge [1] sont souvent le fait d'automutilations chez des patients psychiatriques. Il est également retrouvé d'autres étiologies comme les accidents de la voie publique et les agressions, nécessitant dans tous les cas une bonne prise en charge.

\section{Observation}

M. A.A. âgé de 40 ans, fleuriste de profession, sans antécédents particuliers, est admis en urgence le 23 juillet 2010 pour amputation traumatique de la verge.

L'interrogatoire, peu aisé chez ce patient peu compliant, et l'examen clinique ont permis de noter :

- une notion d'agression physique dans un contexte d'adultère où l'agresseur serait l'époux trompé ; le patient aurait été surpris par l'agression, alors qu'il venait juste d'ouvrir sa porte après que l'époux trompé y eut frappé ;

- une amputation traumatique du phallus à type de section transversale de la verge à $3 \mathrm{~cm}$ environ de sa base avec une plaie hémorragique (Fig. 1) ;

- le segment pénien distal antérieur n'a pas été retrouvé ; 
- l'état général du patient était bien conservé sans perturbation hémodynamique, une tension artérielle à 110/80 mmHg et une conscience conservée ;

- l'absence de plaintes douloureuses qui pourrait s'expliquer par le mutisme dans lequel s'était renfermé le patient;

- l'absence de toute autre lésion physique permettant de déduire qu'il n'y a pas eu de lutte, ce qui est sans doute lié à l'effet de surprise de l'agression.

Il est à noter que le patient a été amené aux urgences par des parents qui l'auraient découvert couché sur son lit gisant dans son sang, ce qui expliquerait un déni par le patient qui serait allé donc se recoucher après l'agression.

Le patient a bénéficié en urgence de :

- hémostase ;

- régularisation du moignon ;

- rapprochement des corps caverneux ;

- mise en place d'une sonde de Foley n ${ }^{\mathrm{o}} 24$;

- suture finale mucocutanée en points de Blair Donati (Fig. 2).

Sur le plan local, les suites opératoires ont été simples. Le patient s'est par ailleurs enfermé dans un mutisme qui a motivé un avis psychiatrique ayant révélé des facteurs dépressiogènes de survenue postérieure à l'agression. Un traitement fait de neuroleptique et d'antidépresseur a été institué. $\mathrm{Au} 20^{\mathrm{e}}$ jour postopératoire, une bonne cicatrisation du moignon est obtenue et on procède à l'ablation de la sonde vésicale. Le patient a bénéficié d'un suivi psychiatrique régulier, et l'évolution sur le plan comportemental est marquée par une amélioration communicationnelle. Le patient

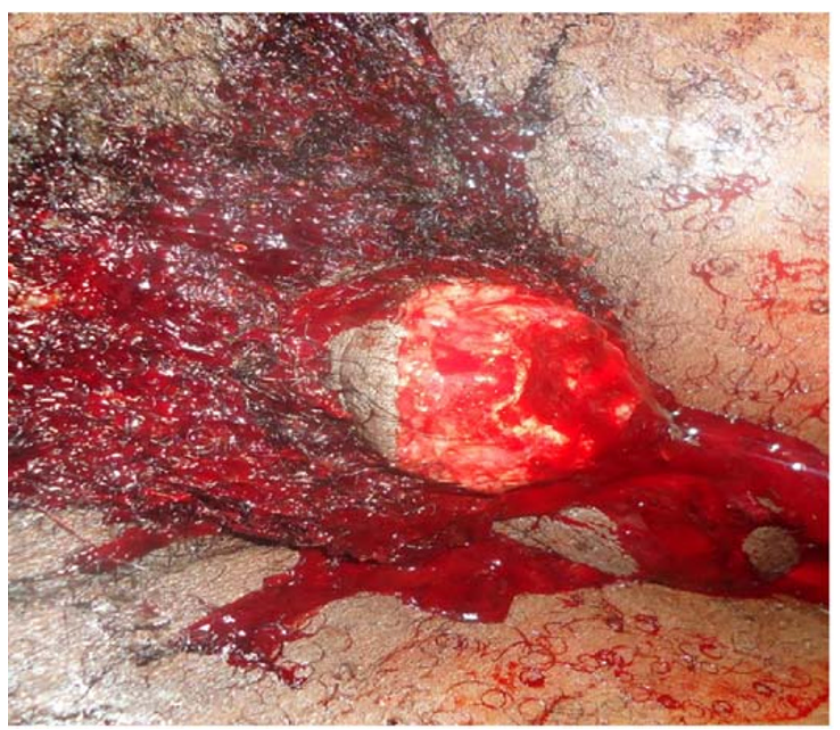

Fig. 1 Plaie de section de la verge à $3 \mathrm{~cm}$ environ de sa base n'a cependant posé aucune question sur ses possibilités sexuelles.

Le patient s'est enfui au $30^{\mathrm{e}}$ jour d'hospitalisation et toutes nos tentatives pour le retrouver furent vaines.

\section{Discussion}

Le pénis de l'homme peut être le siège de traumatismes d'étiologies et de mécanismes variés. Excepté les traumatismes par accident de la voie publique, lorsque survient une amputation pénienne, il est habituel de rechercher un terrain psychiatrique. Dans pareil cas, la prise en charge implique non seulement le chirurgien mais aussi le psychiatre.

En effet, l'amputation traumatique de la verge est très rare [1-3]. Elle est retrouvée dans un contexte d'automutilation chez des patients psychiatriques [2-4]. Dans le cas présenté, il s'agit de deux amis dont l'un serait l'amant de l'épouse de l'autre. Se sentant outragé, l'époux concerné s'est présenté chez son « ami » et lui a amputé la verge par un outil tranchant. Les cas d'amputations " internes » au couple sont signalées dans la littérature : les cas d'amputations péniennes par des épouses déshonorées existent sous forme d'épidémies de violences conjugales en Thaillande [2].

La prise en charge chirurgicale adéquate des amputations péniennes comporte non seulement la régularisation et l'hémostase du moignon pour sauver la vie de la victime mais surtout la " reposition ». La prise en charge thérapeutique relève à la fois d'un bon niveau de développement du plateau technique mais aussi de l'expérience de l'urologue ou du chirurgien dans ce domaine. La reposition consiste en une réimplantation qui doit être tentée chaque fois que cela est possible. Il y a deux situations à considérer : réimplanter avec succès la portion pénienne amputée ou ne pouvoir que régulariser et assurer l'hémostase du moignon d'amputation pénienne. Dans le cas rapporté ici, la prise en charge du patient a consisté en une chirurgie d'hémostase et de régularisation de moignon en urgence, la portion emportée par la section n'ayant pas été retrouvée. Comme le souligne Kochakarn, la réimplantation s'applique à tous les cas où la portion amputée est disponible et non complètement détruite [2]. La réimplantation doit être immédiate ou retardée avec conservation de la portion amputée en congélation pendant 16 à 24 heures [5].

Le volet psychiatrique de la prise en charge psychiatrique en cas d'automutilation pénienne ou d'hétéromutilation a pour fondement la perte de l'intégrité pénienne. Dans ce cas, celle-ci met en jeu le pronostic sexuel et le pronostic esthétique. M. A.A. a bénéficié d'une consultation psychiatrique qui a révélé une note dépressive, alors que dans le cas d'automutilation, c'est une note schizophrénique qui est retrouvée. Dans tous les cas, Kochakarn [2] suggère une évaluation et un accompagnement psychiatrique pour ces patients. 


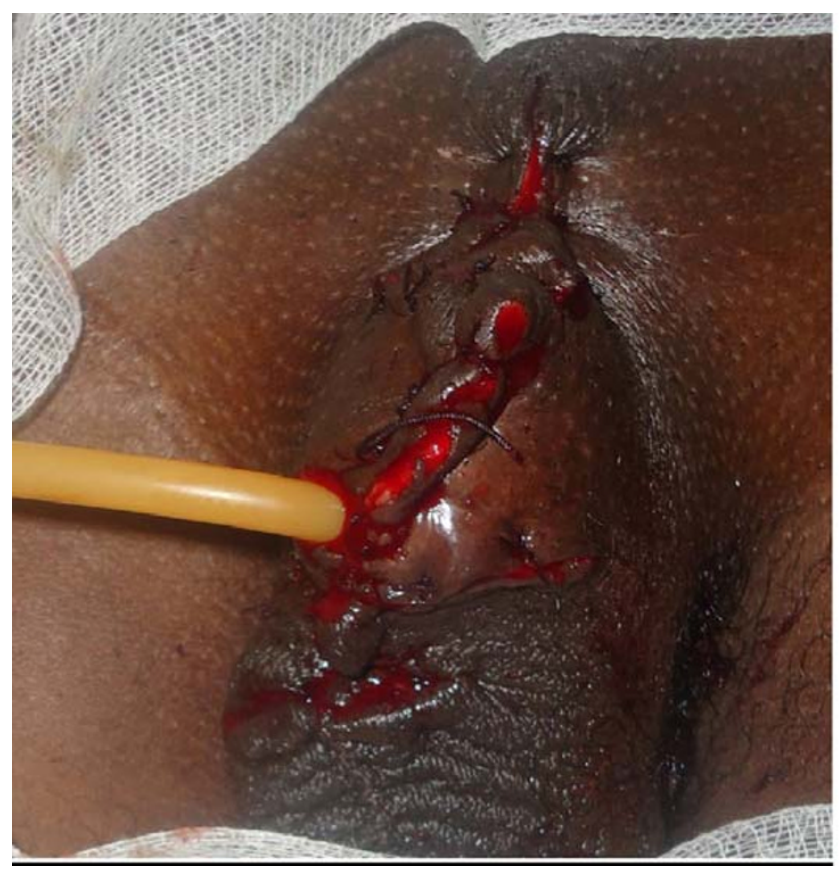

Fig. 2 Moignon après hémostase et régularisation

L'évasion ou la fugue de M. A.A., alors qu'il était apparu une nette amélioration clinique et communicationnelle obtenue avec le début d'accompagnement psychiatrique, a constitué un handicap à la mise en œuvre totale de ce que préconise Kochakarn [2]. Le patient poserait un problème de verge très courte auquel divers procédés d'allongement $\mathrm{du}$ pénis ou de greffe de pénis auraient pu répondre.

Le succès de la réimplantation de la portion amputée ne peut être envisagé sans l'éducation sanitaire des populations à l'acquisition de réflexes de ramassage, d'emballage dans un milieu ou linge propre de la portion amputée à transporter sans délai dans un centre médicotechnique de désinfection et conservation par congélation. Alors seulement le recours à un acte chirurgical spécialisé dans la réimplantation du segment pénien amputé apportera des chances de réussite.

\section{Conclusion}

Les amputations péniennes, qu'elles surviennent par automutilation ou par violences conjugales internes ou violences externes, relèvent d'une prise en charge bidisciplinaire dans laquelle collaborent urologue et psychiatre.

Conflit d'intérêt Les auteurs déclarent ne pas avoir de conflit d'intérêt.

\section{Références}

1. Ahmed A, Mbibu NH (2008) Aetiology and management of injuries male external genitalia in Nigeria. Injury 39:128-33

2. Kochakarn W (2000) Traumatic amputation of the penis. Braz J Urol 26:385-9

3. Sow Y, Fall PA, Diao B, et al (2008) Les traumatismes de la verge : à propos de 23 cas. Andrologie 18:210-5

4. Kabore FA, Fall PA, Diao B, et al (2008) Autoamputation récidivante du pénis sur terrain schizophrène: à propos d'un cas. Andrologie 18:224-6

5. Salem HK, Mostafa T (2009) Primary anastomosis of the traumatically amputated penis. Andrologia 41:264-7 